\title{
Presentation of a new magnetic field therapy system for the treatment of human solid tumors with magnetic fluid hyperthermia
}

\author{
Andreas Jordan ${ }^{\mathrm{a}, *}$, Regina Scholz ${ }^{\mathrm{a}}$, Klaus Maier-Hauff ${ }^{\mathrm{b}}$, Manfred Johannsen ${ }^{\mathrm{c}}$, \\ Peter Wust ${ }^{\mathrm{a}}$, Jacek Nadobny ${ }^{\mathrm{a}}$, Hermann Schirra ${ }^{\mathrm{d}}$, Helmut Schmidt ${ }^{\mathrm{d}}$, Serdar Deger ${ }^{\mathrm{c}}$, \\ Stefan Loening, Wolfgang Lanksch ${ }^{\mathrm{b}}$, Roland Felix ${ }^{\mathrm{a}}$ \\ ${ }^{a}$ Department of Radiation Oncology, Clinic of Radiology, CVK, Campus Virchow-Klinikum, Augustenburger Platz 1, 13353 Berlin, Germany \\ ${ }^{\mathrm{b}}$ Department of Neurosurgery, Clinic of Neurology and Neurosurgery, CVK, Campus Virchow-Klinikum, Augustenburger Platz 1, \\ 13353 Berlin, Germany \\ ${ }^{\circ}$ Clinic of Urology, CCM: Campus Charité-Mitte, Schumannstr. 20/21, 10117 Berlin, Germany \\ ${ }^{\mathrm{d} I n s t i t u t e}$ of New Materials (INM), Im Stadtwald, 66123 Saarbrücken, Germany
}

\begin{abstract}
Magnetic fluid hyperthermia (MFH) selectively heats up tissue by coupling alternating current (AC) magnetic fields to targeted magnetic fluids, so that boundaries of different conductive tissues do not interfere with power absorption. In this paper, a new AC magnetic field therapy system for clinical application of MFH is described. With optimized magnetic nanoparticle preparations it will be used for target-specific glioblastoma and prostate carcinoma therapy.
\end{abstract}

Keywords: Interstitial hyperthermia; Brain tumor; Prostate carcinoma; MFH-applicator; Hyperthermia; AC magnetic field; Magnetic nanoparticle; Glioblastoma

\section{Introduction and state-of-the-art}

Hyperthermia intensifies the efficacy of radiation and/or chemotherapy. Due to technical and physical limitations, some regions of the body cannot be heated adequately with currently available hyperthermia systems or are only accessible by traumatic and highly invasive interstitial methods, e.g., like with brain tumors and locally advanced prostate carcinomas.

\footnotetext{
*Corresponding author. Tel.: + 49-30-45057073; fax: + 4930-45078979.

E-mail address: andreas.jordan@charite.de (A. Jordan).
}

There has been much interest in using thermoradiotherapy in the treatment of primary brain tumors because patients treated conventionally have a very poor prognosis despite a large variety of therapeutic strategies and concepts [1]. The extent of resection is an important prognostic factor for survival [2]. Poorly circumscribed are malignant brain tumors, e.g., anaplastic astrocytomas or glioblastomas. Recurrent tumor growth, i.e., loss of local control at the primary site is the main cause of therapeutic failures even after aggressive treatments like interstitial brain implant boost [3]. Many years ago, it was shown that hyperthermia greatly enhances cytotoxicity of radiation and drug 
treatment with brain tumor cell lines, which were also confirmed by multimodal hyperthermia studies with rats, rabbits and dogs [4]. Toxicity studies revealed a maximum tolerable thermal dose of normal brain in dogs to be $44^{\circ} \mathrm{C}, 30 \mathrm{~min}$, using interstitial microwave antennas. Known side effects of hyperthermia in animal experiments are cerebral necrosis, edema, focal hemorrhage and infarction. A breakdown of the blood-brain barrier is observed at temperatures of $42.5^{\circ} \mathrm{C}-43^{\circ} \mathrm{C}, 60 \mathrm{~min}$. Clinical studies performed so far have shown that interstitial brain hyperthermia is feasible and that toxicity is acceptable under careful control of the heating and limitation of the target volume. In a recently published randomized phase II/III study, 112 patients with glioblastoma multiformae (GM) received a combination of brachytherapy boost \pm interstitial hyperthermia [5]. The results of this important study clearly demonstrate that adjuvant interstitial brain hyperthermia given before and after brachytherapy boost, after conventional radiotherapy, significantly improved GM patient survival (2 year survival $31 \%$ versus $15 \%$ ) with acceptable toxicity. Heating was performed with interstitial microwave antennas $(950 \mathrm{MHz})$, which were implanted $1.2-1.8 \mathrm{~cm}$ apart within the tumor. The power was manually controlled, so that the tumor temperature did not exceed $50^{\circ} \mathrm{C}$ and $44^{\circ} \mathrm{C}$ in the normal brain tissue. Toxicities were more frequent in the hyperthermia arm combined with mild neurological changes and seizures (lasting $\leqslant 5 \mathrm{~min}$ ), which were most probably caused by the overheating of normal brain tissue. Neither problems with edema and intracranial pressure nor infarction or thrombosis was reported in this study. Our own results with the same method using microwave antennas operating at $434 \mathrm{MHz}$ support these encouraging findings [6-9]. In conclusion, the present preclinical and clinical data show that hyperthermia is feasible and effective in combination with radiation therapy. However, in the clinical situation, temperature homogeneity is still the major limiting factor for the therapeutic outcome, which largely requires improvements of intracranial hyperthermia application methods.

Interstitial hyperthermia also has a potential in the treatment of locally advanced prostate carcinomas as an adjunct to interstitial and conformal external beam radiation therapy [10]. The utilization of interstitial radioactive seeds yields significantly higher doses of radiation (up to $100 \mathrm{~Gy}$ and more). The synergistic behavior of hyperthermia and radiation is a well-known phenomenon so interstitial heating techniques also have been developed for the treatment of prostatic tumors. Besides radio-frequency ( $\mathrm{RF}-$ ), microwave and ultrasound applicators, self-regulating thermoseeds have been used [11,12], which are heated by an externally applied AC magnetic field $(25-50 \mathrm{kHz}$, $2 \mathrm{kA} / \mathrm{m})$. The special feature of self-regulating thermoseeds arises from the ferromagnetic alloy, which changes from the ferromagnetic to the nonmagnetic state at the so-called Curie point $\left(T_{\mathrm{C}}\right.$, typically at $55^{\circ} \mathrm{C}$ ), which also indicates the temperature, at which eddy current heating decreases drastically. As with other interstitial heating methods, heat sources must be implanted in an array, where each of the antennas or seeds must have a spacing of around $1 \mathrm{~cm}$ to the adjacent source in order to obtain a more or less homogeneous temperature pattern throughout the tumor. Since all seeds have to be implanted exactly perpendicular to the $H_{0}$ field main axis, a limiting factor is the migration of the seeds, which causes a decrease of the specific power absorption (SAR) and in these cases thermal underdosage [13]. In most of the studies toxicity was acceptable (grade I/II). In our ongoing phase II study for locally advanced prostate carcinomas, interstitial thermoseed hyperthermia plus conformal radiotherapy has indicated good tolerability so far and no acute complications after thermoseed hyperthermia. Since July 1997 more than 50 patients have been treated. Intraprostatic temperatures were within the effective range, i.e., between $42^{\circ} \mathrm{C}$ and $46^{\circ} \mathrm{C}$, whereas in the urethra $38^{\circ} \mathrm{C}-43^{\circ} \mathrm{C}$ were measured. The PSA values decreased from $11.6 \mathrm{ng} / \mathrm{ml}$ (initial) to $0.30 \mathrm{ng} / \mathrm{ml}$ within 1 year after therapy.

\section{Magnetic fluid hyperthermia (MFH): rationale and perspectives}

MFH is a completely new approach for deeptissue hyperthermia application because it couples the energy magnetically to nanoparticles in the 
target region, so that bone or boundaries of different conductive tissues do not interfere with power absorption as with E-field dominant systems used for e.g., regional hyperthermia [14,15]. A further potential arises with magnetic nanoparticles we generated for certain types of cancer, e.g., glioblastoma and prostate carcinoma cells, which are taken up selectively by malignant cells but not by normal cells of the same tissue type.

As it is typical for interstitial antennas or other 'hot sources' like thermoseeds, the tissue is exclusively heated by thermal conduction. Therefore the temperature homogeneity and the minimum tumor temperature depend strongly on the spacing of heating elements (typically $\leqslant 1 \mathrm{~cm}$ ). Taking both together, biological selectivity and a much larger number (lower spacing) of heating elements (coated nanoscaled particles) of a magnetic fluid, one should expect theoretical advantages of biological heat targeting and temperature homogeneity by the $\mathrm{MFH}$, which were missing in the conventional techniques used so far.

Differential endocytosis of modified aminosilan magnetite nanoparticles into primary glioblastoma cells but not in normal glial cells in vitro, has been reported previously [16]. In this study, we observed a 10-fold higher uptake by glioblastoma cells than by normal cells.

Further new aminosilan-type nanoparticle preparations have been manufactured at the Institute of New Materials (INM) Saarbruecken (Germany), which are taken up by prostate carcinoma cells but not by normal prostate cells, endothelial cells or fibroblasts in vitro. Preliminary data indicate that the malignant cells take up nine times more particles than normal cells do. A sample micrograph is shown in Fig. 1, where a fibroblast (F) and a prostate carcinoma cell (ProstCA) are adhering close to each other. The malignant cell exhibits a clearly visible pigmentation due to large nanoparticle uptake in contrast to the adjacent fibroblast. Identification of cell type was performed by immuncytological analysis. Since the prostate carcinoma culture contained $100 \%$ cytokeratin $18^{+}$ and cytokeratin $8^{+}$cells, no normal prostate cells were present in the culture. The culture was in the 5th passage and thawed from frozen stock, so the presence of any macrophages was very unlikely.
Fibroblast marker positive cells were clearly discernible by their morphology typical of the fibroblasts in contrast to the epithelial-type cells of prostate carcinoma.

Since uptake of these particles is also observed into macrophages in vivo (data not shown), a systemic targeting approach is not promising. Methods of the so-called interventional radiology are required to precisely infiltrate the tumor. In the case of brain tumors, conventional stereotactic and navigation systems offer an accurate positioning. Application of $\mathrm{MFH}$ to prostate carcinomas requires ferrofluid administration under sonographic control.

\section{AC magnetic field application: the new clinical MFH therapy system}

Besides biocompatible magnetic nanoparticles stabilized as a magnetic fluid, MFH requires also an AC magnetic field applicator system. Note that physical dimensions and field-frequency parameters must match the limitations given by eddy current heating of the highly conductive tissue as it was calculated earlier [15]. The technical and medical requirements of such an applicator system in terms of e.g., field homogeneity, accuracy of field strength and frequency, safety, choice of treatment volume, thermometry and clinical quality assurance have been largely underestimated so far. In experimental setups to treat small animals, the construction of an AC magnetic field applicator is relatively simple. Both, induction coils and ferromagnetic core-coil constructions can be used as applicators because currents and voltage are moderate and controllable using resonance circuits. In contrast, in a clinical situation a much larger air volume (at least $5-10000 \mathrm{~cm}^{3}$ for regional hyperthermia) has to be 'filled up' with the AC magnetic field, which is a very power consuming approach. On the other hand, large currents and voltages effect electrical and operating safety of the overall system. Field homogeneity is crucial, because each particle has its specific power absorption, which is only constant if the applied field is almost homogeneous. Field gradients yield thermal gradients even when a homogeneous particle distribution has 


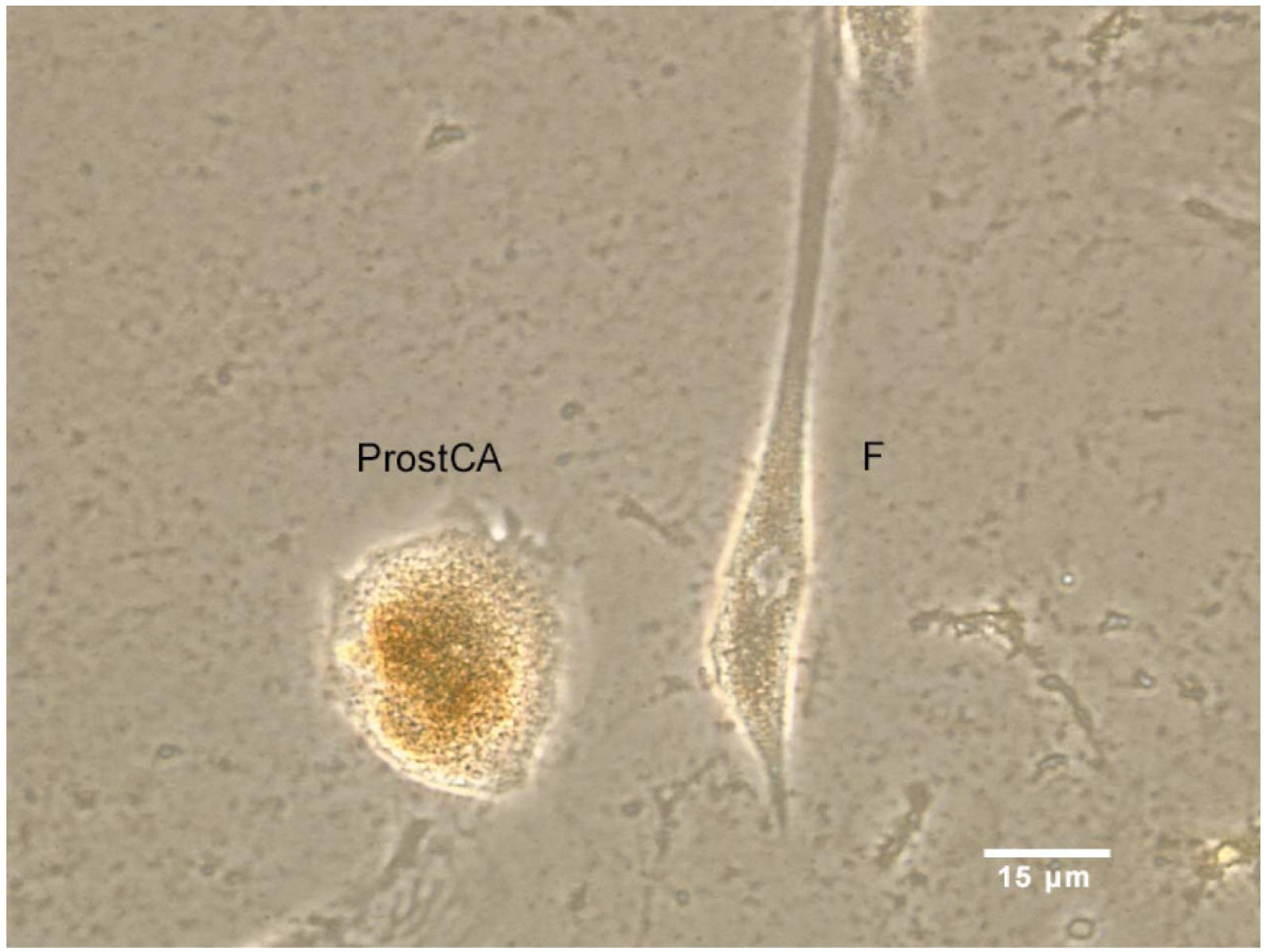

Fig. 1. Phase-contrast light microscopic picture of a prostate carcinoma cell and a fibroblast cell lying adjacent to each other. Whereas the tumor cell shows remarkable pigmentation due to large nanoparticle uptake, the adjacent fibroblast cell depicts lower pigmentation, i.e., no or lower particle uptake. The cell types were determined in separate experiments according to their morphology in combination with immunocytological data.

been achieved. Since it is more difficult to achieve a homogeneous particle distribution within the tissue than in a homogeneous field in air, the field should be as homogeneous as possible. Furthermore, heating of a small amount of nanoparticles (about $10 \mathrm{mg}$ ferrite/g tumor) requires about 5 times higher field amplitudes (in the order of $10 \mathrm{kA} / \mathrm{m}$ ) in comparison to thermoseed heating. For these overall requirements and limitations, inductive coils are not suitable in contrast to thermoseed hyperthermia. Scaling up such a system to the $\mathrm{MFH}$ requirements would be necessarily associated with unacceptable high E-fields, power and heat losses close to the patient and high operating costs. Therefore, a ferrite-core-based applicator system is the only alternative, which can be used for medical MFH application.

By the end of 2000, the first prototype of a clinical MFH therapy system will be set up at the Charite Medical School, Campus VirchowKlinikum, Clinic of Radiation Oncology in Berlin. It is a ferrite-core applicator operating at a frequency of $100 \mathrm{kHz}$ with an adjustable vertical aperture of $30-45 \mathrm{~cm}$. The field strength is adjustable from 0 to $15 \mathrm{kA} / \mathrm{m}$. A sketch of the new system is shown in Fig. 2. The system generates a field, 


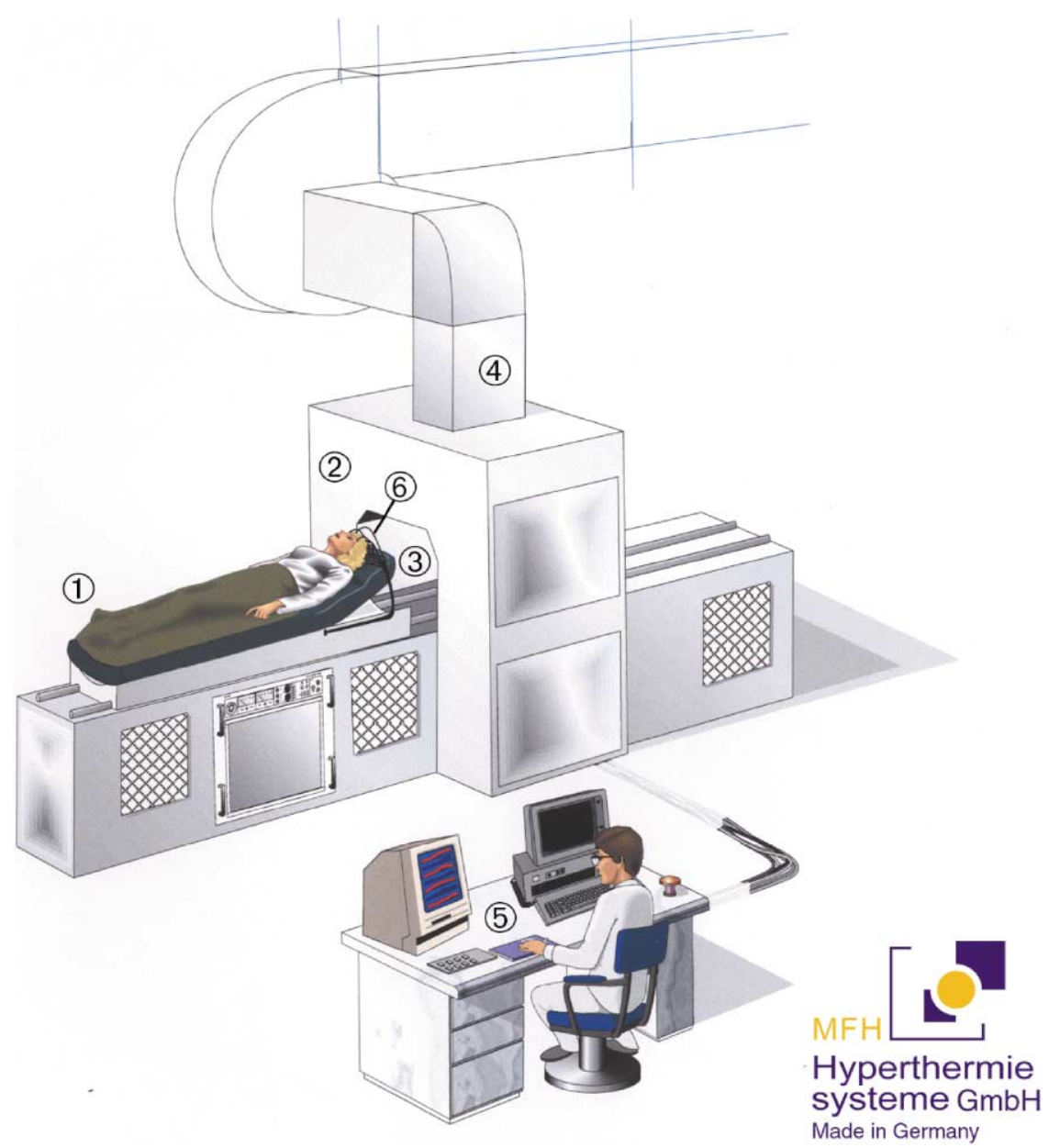

Fig. 2. Sketch of the first prototype MFH therapy system (MFH Hyperthermiesysteme GmbH, Berlin, Germany). The AC magnetic field axis is perpendicular to the axial direction of the patient couch (1). The therapy system is for universal application, i.e., suitable for MFH within, in principle, any body region. It is a ferrite-core applicator (2) operating at a frequency of $100 \mathrm{kHz}$ with an adjustable vertical aperture of $30-50 \mathrm{~cm}$ (3). The field strength is adjustable from 0 to $15 \mathrm{kA} / \mathrm{m}$. The system is air cooled (4). Aperture, field strength, thermometry and further system parameters are on-line monitored and adjusted manually by the physician at the control unit (5). The temperature is measured invasively with fluorooptic temperature probes within the tumor and at reference points outside the patient (6).

perpendicular to the axial direction of the patient couch. The core of the system is air cooled and connected to a heat exchanger located in the ceiling.
The treatment room is shielded and several surveillance systems are implemented for the safety of the physician and the patient. In the foreground, the physician is shown at the terminal, where he

Fig. 3. 3D field strength distributions were calculated using the 'finite-difference-time-domain-(FDTD)-method' for different applicator apertures, currents, geometry of the core and axial positions of the patient. One example of the visualized results is shown in Fig. 3A (3D vector diagram, coronary view, $24 \mathrm{~cm}$ aperture, $15 \mathrm{kA} / \mathrm{m}$ ) and $3 \mathrm{~B}$ (corresponding numerical plot). 
Fig. 3A

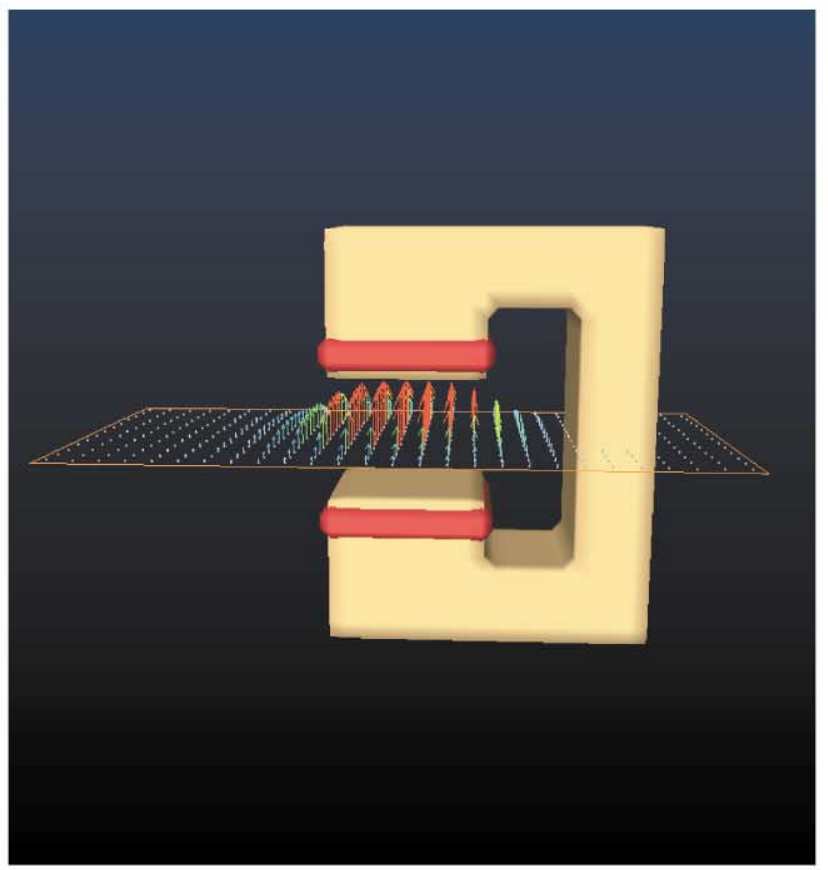

Fig. 3B

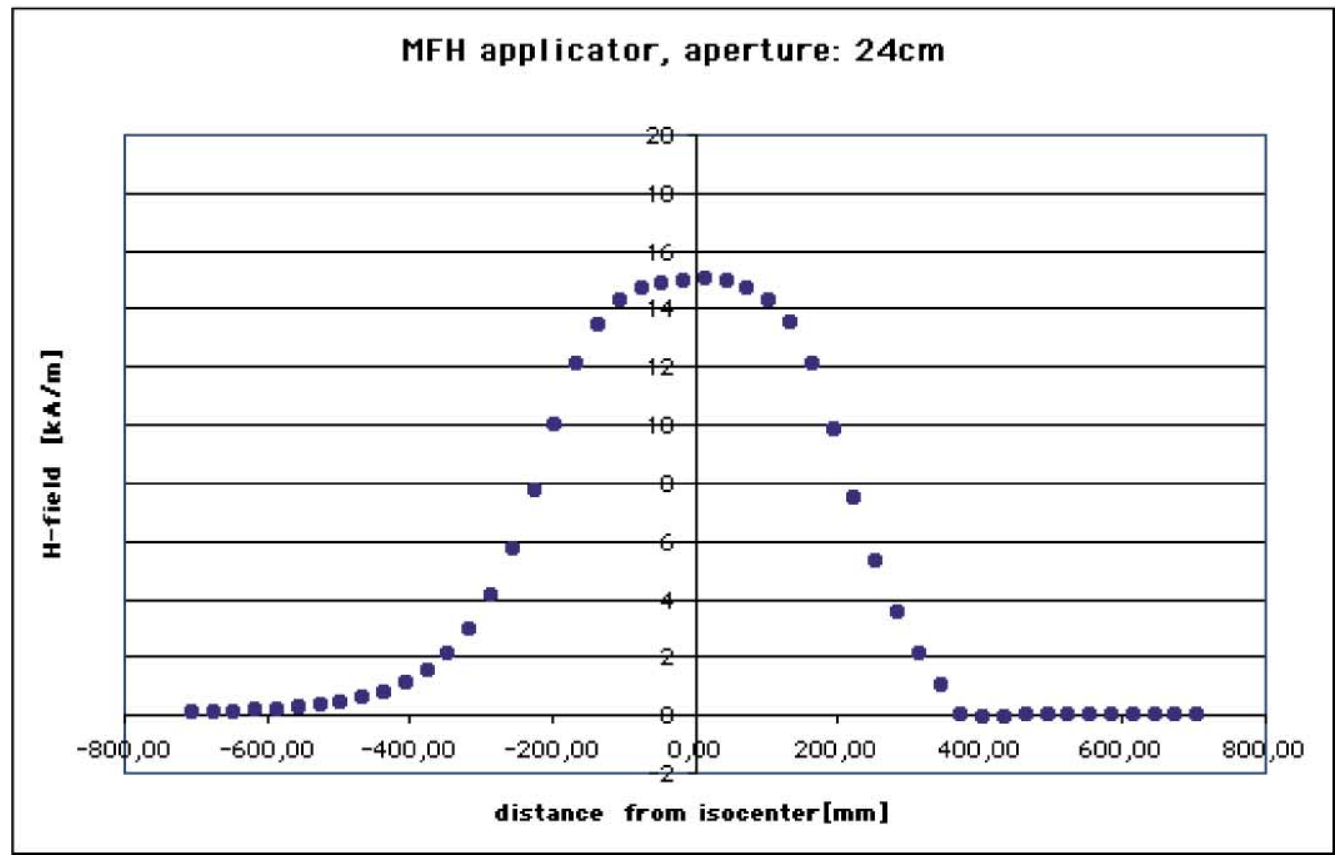


controls the temperature elevations, the magnetic field strength and the surveillance systems. Video cameras are installed for the communication between patient and physician during the therapy course. For all variations of aperture, currents, geometry of the core and axial positions of the patient, the resulting 3D field strength distributions were calculated using the 'finite-difference-time-domain-(FDTD)-method'. One example of the visualized results is shown in Fig. 3A (3D vector diagram) and 3B (numerical plot). The MFH therapy system has been developed for universal application, especially for regional hyperthermia. With this equipment, brain tumors as well as prostate and other cancer entities can be treated very homogeneously with the required $\mathrm{AC}$ magnetic field. The system is equipped with a fluorooptic thermometry with $0.55 \mathrm{~mm}$ diameter single channel probes or $0.9 \mathrm{~mm}$ multi-channel probes (typically four-fold probes with $0.5 \mathrm{~cm}$ spacing) for thermal mapping, so that during AC magnetic field application the temperature can be measured on-line in the target region and at free eligible reference points. The temperature course and further data like power and setup parameters are displayed on a personal computer during therapy. System control and thermometry have been implemented in a new application software. The AC magnetic field strength is manually adjusted to the desired steady-state temperature in the tumor, i.e., the average temperature of at least three target measurement points. The thermometry device is further equipped with a calibration setup unit. The accuracy of the thermoprobes is $\pm 0.3^{\circ} \mathrm{C}$, which is necessary for hyperthermia application in general.

\section{Phase I/ II clinical concept for brain tumors}

Candidates for MFH brain tumor application are circumscribed glioblastomas, brain metastases, residual disease after glioblastoma resection and recurrent brain tumors. Application to the brain implies moderate thermal dosage, i.e., 'classical hyperthermia' at temperatures of $42^{\circ} \mathrm{C}-45^{\circ} \mathrm{C}$, because treatment volumes are larger than in the case of thermal ablation techniques (e.g., laser-induced thermal therapy). Thermal dose escalation is optional depending on toxicity and tolerance of the first MFH therapies. Analogous to other interstitial hyperthermia applications, MFH of brain tumors will be generally performed without anesthesia, because (1) it may interfere with perfusion and metabolic status of the treated tissue and (2) the requirements that the patient is able to communicate any pain or discomfort to the physician during treatment. The experience with conventional regional hyperthermia systems has shown so far that this is an important aid to avoid normal tissue damage. In response to the patient's sensations, the physician can reduce the field power. However, since it is known that thermal dose parameters are clearly correlated with therapeutic outcome, this reduction might also increase the risk of therapeutic failure. Analogous to thermoseed interstitial hyperthermia, patients who have metallic non-removable compounds (e.g., prosthesis, fragments, cardiac pacemaker, artificial hearts) within or near the treatment region must be excluded from the study.

The following strategies are intended with brain tumors: MFH of non-resectable metastasis or limited sized $(\leqslant 3 \mathrm{~cm})$ glioblastomas will be treated by stereotactic implantation of ferrofluid $(15 \mathrm{mg} \mathrm{Fe} / \mathrm{g}$ tumor), 5-6x $\mathrm{MFH}\left(45^{\circ} \mathrm{C}, 30 \mathrm{~min}\right)$ plus conformal external beam radiotherapy $(5 \times 2 \mathrm{~Gy}$, total $60 \mathrm{~Gy}$ plus dose escalation). MFH of residual disease after glioblastoma resection will be done intraoperatively, i.e., as an open biopsy approach: multifocal implantation of ferrofluid into the $1-2 \mathrm{~cm}$ margin of the resection hole, 5-6 $\mathrm{MFH}$ treatments $\left(45^{\circ} \mathrm{C}\right.$, $30 \mathrm{~min})$ in combination with conformal external beam radiotherapy (EBRT: $5 \times 2 \mathrm{~Gy}$, total $60 \mathrm{~Gy}$ plus dose escalation). 30-45 min after ferrofluid application, the AC magnetic field is applied. Hyperthermia treatment is repeated twice a week. A flow chart of this concept is shown in Fig. 4.

The physician prescribes the desired steady-state temperature by regulating the applied field strength. Before each MFH therapy, CT and MRI are used to verify the appropriate distribution of the ferrofluid load. Positron-emission-tomography (PET) is also used routinely at our clinic so that the identification of metabolically active, e.g., residual, tumor tissue can be localized for subsequent ferrofluid applications. Analogous to the results of earlier animal experiments, we expect that the 


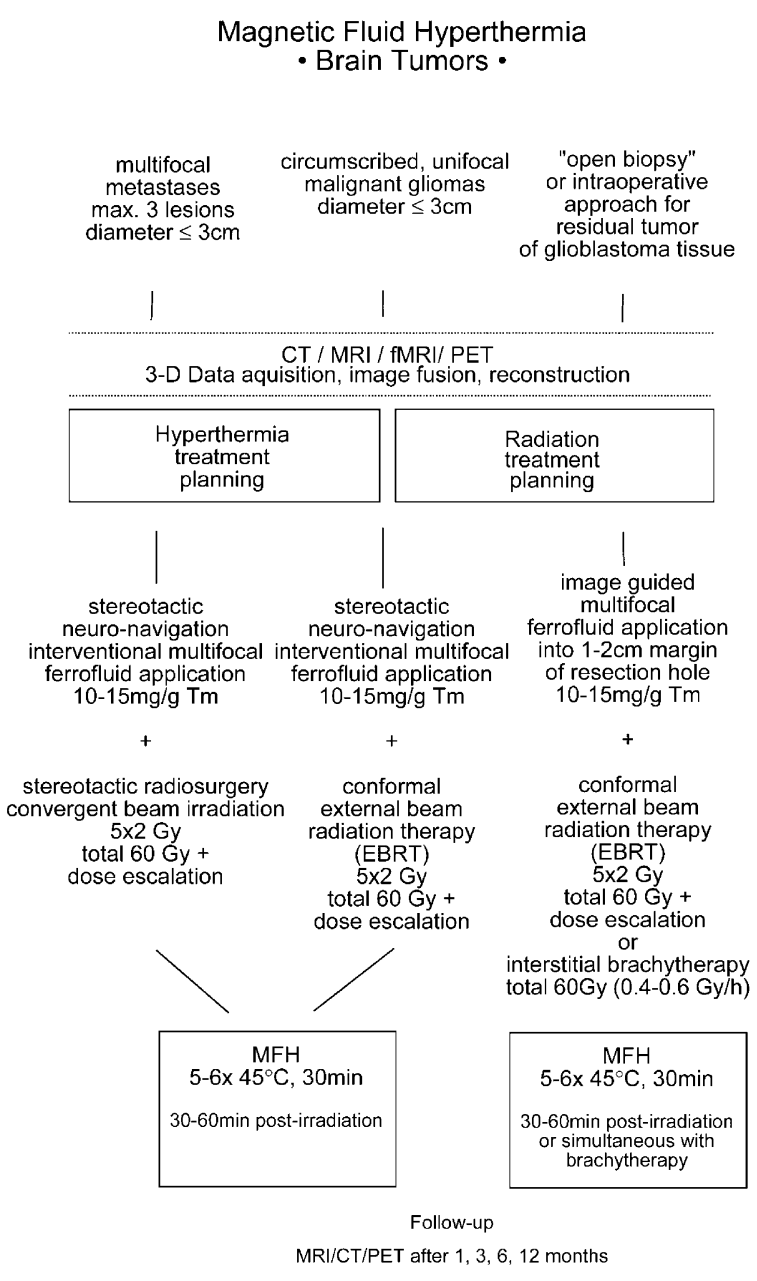

Fig. 4. Concept of a phase I/II study for the treatment of brain tumors with $\mathrm{MFH}$ in combination with conventional radiotherapy (Tm: estimated tumor mass).

'thermal bystander effect' [17] increases step-bystep the homogeneity of the intratumoral nanoparticle distribution and therefore heat deposition throughout the tumor. The heat driven extension of the particle distribution into the critical tumor margin is a desired effect.

\section{MFH for locally advanced prostate carcinomas - a perspective}

The use of interstitial MFH at temperatures of $43^{\circ} \mathrm{C}-47^{\circ} \mathrm{C}$ in thermoradiotherapy of locally ad- vanced carcinoma of the prostate seems very promising. As described above, improvements in thermal homogeneity observed with $\mathrm{MFH}$ are expected to overcome limitations of treatment efficacy due to 'cold spots' within the target volume, which can be encountered using interstitial seeds. A current study confirmed a constant temperature within the prostate [18]. In a clinical setting, transperineal implantation of ferrofluid depots in the prostate can be done under transrectal ultrasound guidance, similar to the technique currently used for thermoseed placement.

Animal experiments with Dunning prostate carcinoma of the rat are in progress to further evaluate the effects of MFH and to establish the optimal treatment temperature. A clinical concept of sequential $\mathrm{MFH}$ as an adjunct to conformal external beam radiation therapy will be formulated soon.

\section{Acknowledgements}

This study was supported by the Deutsche Forschungsgemeinschaft (DFG), Bonn, Germany, SFB 273: 'Hyperthermie: Methodik und Klinik', Project A8.

\section{References}

[1] G.G. Giles, M.F. Gonzales, in: A.H. Kaye, E.R. Laws (Eds.), Brain Tumors, Churchill Livingstone, Edinburgh, 1995.

[2] G.R. Harsh IV, in: A.H. Kaye, E.R. Laws (Eds.), Brain Tumors, Churchill Livingstone, Edinburgh, 1995.

[3] M.W. McDermott, P.K. Sneed, P.H. Gutin, Sem. Surg. Oncol. 14 (1998) 79.

[4] P.K. Sneed, B. Stea, in: M.H. Seegenschmiedt, P. Fessenden, C.C. Vernon (Eds.), Thermoradiotherapy and Thermochemotherapy, Vol. 2, Springer, Berlin, 1996.

[5] P.K. Sneed, P.R. Stauffer, M.W. McDermott et al., Int. J. Radiat. Oncol. Biol. Phys. 40 (1998) 287.

[6] H. Stahl, P. Wust, K. Maier-Hauff et al., Strahlenther. Oncol. 171 (1995) 510.

[7] K. Maier-Hauff, H. Stahl, W. Kluge et al., in: H.U. Lemke et al. (Eds.), CAR'96 Computer Assisted Radiology, Elsevier, Amsterdam, 1996.

[8] K. Maier-Hauff, W. Kluge, H. Stahl, J. Gellermann, P. Wust, Akt. Neurol. 23 (1996) 63.

[9] K. Maier-Hauff, H. Stahl, W. Kluge et al., J. Neurol. 243 (1996) 11. 
[10] F.A. Gibbs, in: M.H. Seegenschmiedt, P. Fessenden, C.C. Vernon (Eds.), Thermoradiotherapy and Thermochemotherapy, Vol. 2, Clinical Applications of Hyperthermia, Springer, Berlin, 1996.

[11] I.A. Brezovich, R.F. Meredith, Radiol. Clin. North Am. 27 (1989) 589.

[12] R.D. Tucker, T. Ehrenstein, S.A. Loening, in: D. Schnorr, S.A. Loening, S. Dinges, V. Budach (Eds.), Blackwell, Berlin, 1995.

[13] I.A. Brezovich, M.B. Lilly, R.F. Meredith, Int. J. Hyperthermia 6 (1990) 117.

[14] P. Wust, M. Seebass, J. Nadobny, R. Felix, in: M.H. Seegenschmiedt, P. Fessenden, C.C. Vernon (Eds.), Thermo- radiotherapy and Thermochemotherapy, Vol. 1, Biology, Physiology, and Physics, Springer, Berlin, 1996.

[15] A. Jordan, P. Wust, H. Fähling et al., Int. J. Hyperthermia 9 (1993) 51.

[16] A. Jordan, R. Scholz, P. Wust et al., J. Magn. Magn. Mater. 194 (1999) 185.

[17] A. Jordan, P. Wust, R. Scholz et al., Int. J. Hyperthermia 13 (1997) 587.

[18] J.A. Paulus, R.D. Tucker, S.A. Loening et al., J. Endourol. 11 (1997) 295. 